\title{
DRGs in Transfusion Medicine and Hemotherapy in Germany
}

\author{
Matthäus Bauer $^{\mathrm{a}}$ Helmut Ostermann ${ }^{\mathrm{b}}$ \\ ${ }^{\text {a }}$ Department of Medical Management, \\ ${ }^{\mathrm{b}}$ Department of Haematology, Oncology, University of Munich, Grosshadern, Munich, Germany
}

\section{Keywords \\ DRGs · Transfusion}

\section{Summary}

Patients requiring transfusion medicine and hemotherapy in an inpatient setting are incorporated into the German Diagnosis Related Groups (G-DRG) system in multiple ways. Different DRGs exist in Major Diagnostic Category 16 for patients that have been admitted for the treatment of a condition from the field of transfusion medicine. However, the reimbursement might be not cost covering for many cases, and efforts have to be intensified to find adequate definitions and prices. We believe that this can only be successful if health service research is intensified in this field. For patients requiring hemotherapy and transfusion medicine concomitant to the treatment of an underlying disease such as cancer, multiple systems exist to increase remuneration, among them the Patient Clinical Complexity Level (PCCL) and complex constellations to induce DRG splits. For direct reimbursement of high cost products, additional remuneration fees (Zusatzentgelte, ZE) are the most important. In addition, expensive innovations not reflected within the DRGs can be reimbursed after application and negotiation of the New Diagnostic and Treatment Methods (Neue Untersuchungs- und Behandlungsmethoden, NUB) system. The NUB system guarantees that medical progress is put rapidly into clinical practice and prevents financial issues from becoming a stumbling block for the use of innovative drugs and methods.

\author{
Schlüsselwörter \\ DRGs · Transfusion
}

\section{Zusammenfassung}

Patienten, die eine stationäre transfusionsmedizinische und hämotherapeutische Behandlung erfordern, sind in verschiedener Weise in das System der Diagnosebezogenen Fallgruppen (German Diagnosis Related Groups, GDRGs) inkorporiert. Die Major Diagnostic Category 16 enthält verschiedene DRGs für Patienten, die zur Behandlung einer Erkrankung aus dem Bereich der Transfusionsmedizin stationär aufgenommen werden. In vielen Fällen deckt die Kostenerstattung jedoch nicht die eigentlichen Kosten, weshalb verstärkt Anstrengungen unternommen werden müssen, adäquate Definitionen und Preise festzulegen. Wir sind der Meinung, dass dies nur auf der Basis einer intensiveren Forschung auf diesem Gebiet möglich ist. Für Patienten, die hämotherapeutische und transfusionsmedizinische Maßnahmen begleitend zur Behandlung der zugrunde liegenden Erkrankung (z.B. Krebs) benötigen, existieren verschiedene Systeme zur Aufstockung der Kostenerstattung. Dazu gehören der Patient Clinical Complexity Level (PCCL) sowie komplexe Konstellationen, die DRG-Splits zulassen. Für die direkte Erstattung von Kosten für sehr teure Produkte sind vor allem die Zusatzentgelte (ZE) bedeutsam. Zusätzlich können kostenaufwendige Innovationen, die nicht in den DRGs enthalten sind, durch Anwendung und Übertragung des NUB(Neue Untersuchungs- und Behandlungsmethoden)-Systems gedeckt werden. Das NUB-System stellt sicher, dass medizinischer Fortschritt rasch in die klinische Praxis übertragen wird, und verhindert, dass finanzielle Fragestellungen die Anwendung innovativer Medikamente und Methoden behindern.

\section{KARGER \\ Fax +497614520714 \\ Information@Karger.de}

www.karger.com (c) 2012 S. Karger GmbH, Freiburg

$1660-3796 / 12 / 0392-0060 \$ 38.00 / 0$

Accessible online at:

www.karger.com/tmb
Dr. Matthäus Bauer, MBA

Ref. VII.2 für Medizinisches Leistungsmanagement

Klinikum der Universität München

81366 München, Germany

Tel. +49 89 709560-38, Fax -39

mbauer@med.lmu.de 


\section{Introduction}

In modern medicine, blood and its components have become an indispensable product widely used in patients undergoing surgery, chemotherapy, transplantation, or obstetric and gynaecological procedures [1]. Among all German hospitals, more than 925,000 blood transfusions were documented in 2007, and plasma products were used in more than 191,000 cases [2]. In 2008, the numbers were 968,000 and 205,000, respectively. Approximately $92-95 \%$ of blood is transfused in the inpatient setting [3]. Shrinking donor pools and stringent donor qualification criteria can lead to rising costs and constraints in the supply of blood products [4]. Although in general only about $1 \%$ of the total inpatient treatment costs are caused by hemotherapy, this figure can substantially rise in patients requiring frequent transfusions such as in leukaemia treatment, organ transplantation, or obstetrics [5]. Therefore, it is important for hospitals to focus on optimal blood acquisition and to avoid inappropriate blood transfusions [6]. Negotiating adequate reimbursement for transfusions is also a major concern for hospitals.

In Germany, the German Diagnosis Related Group (GDRG) lump sum system was introduced in 2003, and is updated annually. This system was designed to be based on real cost data and to be self-adapting. All 2,087 German hospitals are obliged to transmit clinical data on all inpatient cases to the National Institute for the Hospital Remuneration System (Institut für das Entgeltsystem im Krankenhaus, InEK). Data include diagnoses, services, basic patient data, and the duration of hospitalization. Apart from this mandatory part, hospitals have the option to supply accounting data. For the calculation of the 2010 G-DRG system, 253 hospitals provided data on 4.8 million cases, which included detailed information on the number of blood products transfused as well as accurate cost data, in particular costs of each blood product. G-DRG could best be compared to Medicare's prospective payment system in the USA where also an annual process for updating exists which includes the pricing of the hospital market basket and the weighting of the DRGs $[3,7,8]$.

This review focuses on the 2010 G-DRG system and addresses issues that are specific to hemotherapy: What tools do we need for adequate mapping of the costs in a lump sum system? How can we try to adequately remunerate the treatment of a patient presenting with a diagnosis typical for transfusion medicine? How can we try to adequately remunerate the treatment of all patients presenting with diagnoses typical for transfusion medicine?

\section{Lump Sums and Additional Remuneration Fees Are the Backbone of the G-DRG System}

The G-DRG system is mainly based on lump sums. It was developed on the basis of the Australian AR-DRG system ver-

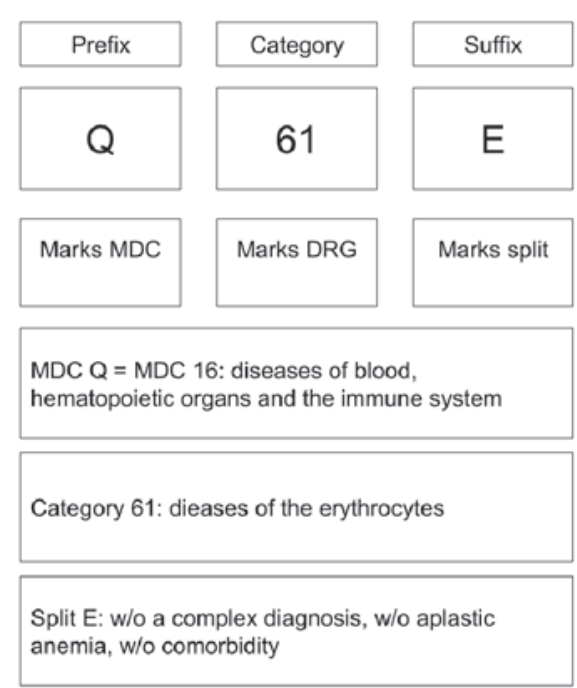

sion 4.1, but has since continuously matured [9]. Today, with the 7 th amendment in place, only the nomenclature reminds of the Australian predecessor: Alphabetic prefixes from A to $\mathrm{Z}$ mark the 24 Major Diagnostic Categories (MDC). These are categorized mainly by the anatomical location of the disease. Numbers from 00 to 99 address the DRG itself. Suffixes differentiate between DRG splits, with A usually representing the most expensive DRG. The alphabetic sequence of suffixes is variable and can reach counts from 1 (usually marked with Z) to 9 (from A down to I).

The DRG finding process is automated, and only approved Grouper software is authorized. The MDC is mainly triggered by the so-called principal diagnosis. The principal diagnosis is defined in official guidelines as the diagnosis identified to be mainly responsible for the hospitalization of the patient [10]. The diagnosis is coded by use of the WHO International Classification of Diseases (ICD-10), German modification (ICD10 GM) [11].

Interventional services, and surgeries in particular, mainly push the case into more expensive DRGs within a MDC. Among the medical services, the DRGs are mainly split up by expenditures. Comorbidity is the major criteria to lead a case into a more expensive DRG split. Figure 1 shows the basic principle of the G-DRG system based on the example of DRG Q61E which is the most common DRG for patients being hospitalized for anaemia.

DRGs are temporary, and so-called lower and upper trim points are defined for each DRG. Between the 2 trim points, a lump sum is paid regardless of the length of the hospital stay. Below the lower trim point, diurnal deductions have to be accepted. Beyond the upper trim point, per diem rates are added to the DRG lump sum remuneration, and again the amount of the per diem rate is DRG-specific (fig. 2).

In 2005, with the introduction of the 2 nd amendment of the G-DRG system, the authorities realized that a lump sum alone does not sufficiently cover expenses arising in patients 
Fig. 2. Typical progression of DRG lump sum remuneration dependent on the length of hospitalization; G-DRG 2010, base rate EUR 2,935.00 (DRG Q61E. UGVD = Lower trim point (full DRG is paid after a 2-night stay); OGVD = upper

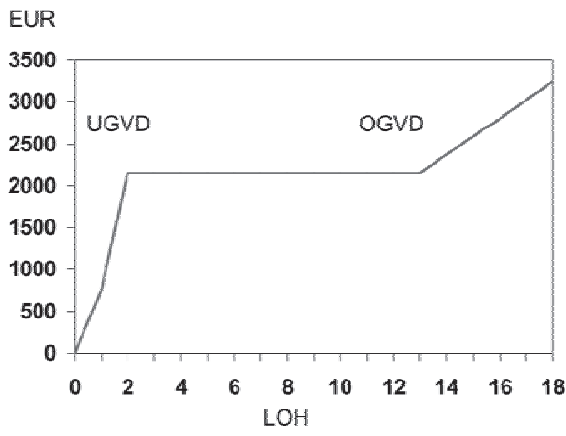

trim point (a per diem rate is paid for the 13th night and beyond); $\mathrm{LOH}$ $=$ length of hospitalization (nights))

Table 1. Additional remuneration fees (ZE) for packed red cells (PRC) in the 2010 G-DRG system

\begin{tabular}{cc}
\hline PRC transfusions, $\mathrm{n}$ & Remuneration, EUR \\
\hline $0-5$ & 0.00 \\
$6-10^{\mathrm{a}}$ & 608.10 \\
$11-15^{\mathrm{a}}$ & $1,004.60$ \\
$16-23$ & $1,480.50$ \\
$24-31$ & $2,115.00$ \\
$32-39$ & $2,749.40$ \\
$40-47$ & $3,383.90$ \\
$48-55$ & $4,018.40$ \\
$56-63$ & $4,652.90$ \\
$64-71$ & $5,287.40$ \\
$72-79$ & $5,921.90$ \\
$80-87$ & $6,556.40$ \\
$88-103$ & $7,402.30$ \\
$104-119$ & $8,671.30$ \\
$120-135$ & $9,940.30$ \\
$136-151$ & $11,209.30$ \\
$152-167$ & $12,478.20$ \\
$168-183$ & $13,747.20$ \\
$184-199$ & $15,016.20$ \\
$200-215$ & $16,285.10$ \\
$216-231$ & $17,554.10$ \\
$232-247$ & $18,823.10$ \\
$248-263$ & $20,092.10$ \\
$264-279$ & $21,361.00$ \\
280 and more & $22,630.00$ \\
\hline${ }^{a}$ Only for children below the age of 15 years. \\
\hline
\end{tabular}

who require components so expensive that the costs far exceed those usually found among a particular group of patients. This observation led to the introduction of additional remuneration fees (Zusatzentgelte, ZE) for components or services. The $\mathrm{ZE}$ are not designed to recover all acquisition costs for hemotherapy. The aim was to design an 'airbag system' capable to hedge extreme costs. Table 1 displays the fees paid for the transfusion of packed red cells (PRC). No fee is paid for patients receiving 1-5 PRC transfusions, hence it is assumed that the costs are covered by the DRG lump sum remuneration itself. Also no fee is paid for adolescents over 14 years of age and adults receiving 6-15 transfusions. A calculation of the per unit remuneration in table 1 shows that the average remuneration for 1 PRC in the G-DRG system depending on the rank within the table is around EUR 75.00 per unit. This, however, applies only to adult patients receiving 16 or more transfusions. Therefore, costs for the first 15 PRC transfusions have to be covered within the respective DRG. At the same time, it is very likely that the remuneration for PRC transfusions in the G-DRG system does not cover the costs. Per unit blood transfusion costs shown in systemic studies $[12,13]$ ranged from USD 332.00 to USD 717.00 (adjusted for inflation), and newer studies say that even this price is inaccurate and misleading [14]. Newer activity-based analyses show that blood costs have been underestimated when only acquisition costs were taken into account. Shander et al. [14] calculated in a model considering all major process steps that 3.2-4.8-fold higher costs emerge (estimated RBC unit costs USD 761.00 \pm USD 294.00). Since the G-DRG cost calculations are based on real life costs from hospital accounting systems, the most plausible conclusion is that cost updates are flawed by the poor quality of hospital cost data. The same problem was observed in the USA where only $48 \%$ of the hospitals billed at all for blood-related costs in their cost report for Medicare [3]. These numbers clearly show that DRG remuneration will only be as good as the cost data provided. Hospitals must aspire to provide complete data, taking into account all costs associated with transfusion (not only acquisition costs), since this is the only way the DRG system can be optimized [15].

\section{Challenge 1: Trying to Adequately Remunerate the Treatment of a Patient Presenting with a Principal Diagnosis Typical for Transfusion Medicine}

The G-DRGs for hemotherapy and transfusion medicine have not changed significantly over time. A comparison of the DRGs in the MDC 16 (diseases of the blood, haematopoietic organs, and the immune system) shows that while during the 8 years of developing the G-DRG system the overall number of DRGs has increased considerably, the number of DRGs in MDC 16 has risen only modestly. G-DRG 2003 (version 1.0) was composed of 664 DRGs, among them 10 DRGs in MDC 16. 8 years later, G-DRG 2010 consists of 1,200 DRGs, and in MDC 16 a total of 16 DRGs can be found. The addition of almost 550 DRGs was done to assure adequate recovery of costs for most of patients, especially those with extremely high costs. Why MDC 16 was not extended to the same degree, may have 1 of 2 reasons: Either the quality of remuneration in MDC 16 was so good that there was no need for an adaptation, or the quality of the data used for calculation was so bad that the authorities could not find enough characteristic factors to differentiate the DRGs in another way. 


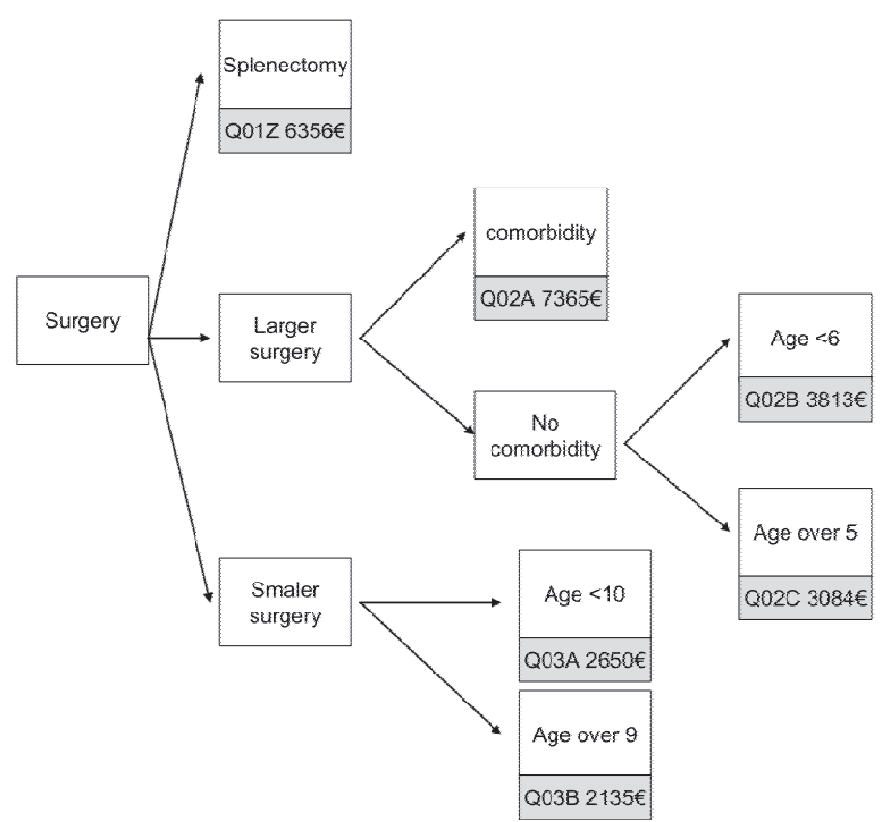

Fig. 3. MDC 16 - Surgical DRGs, simplified (base rate EUR 2,935.00).

The G-DRG 2010 System Has 4 Central Trigger Points to Find the Correct DRG in MDC 16

Surgery in patients with a principal diagnosis eligible for MDC 16 is split up into 3 groups: splenectomy (DRG Q01), major surgery (DRG Q02), and minor surgery (DRG Q03) (fig. 3). Erythrocyte diseases are separated (DRG Q61) from the diseases of the immune system and coagulation disorders (DRG Q60). In both DRG Q60 and Q61, special diagnoses qualify for a more expensive DRG. In all mentioned DRGs, children are treated separately.

Figure 4 visualizes the algorithm for the erythrocyte diseases. Complex diagnoses are been guided to DRG Q61B always. Only few diagnoses have been classified as being complex (table 2). Interestingly, further comorbidity is of no relevance in patients with a complex diagnosis, and regardless of the comorbidity level (see also definition in the lower sections) all patients with complex diagnoses are always grouped to DRG Q61B. The second subgroup are patients with aplastic anaemia (ICD-10 D61.-), and again all patients with this diagnosis are grouped to DRG Q61C or Q61D, respectively, without any focus on comorbidity. This results in a paradox: an adult patient with aplastic anaemia and a very high level of comorbidity is always allocated to DRG Q61D (revenue EUR 2,478.00) whereas EUR 3,680.00 would be paid if another type of anaemia was described. This paradox is an indicator for the immaturity of the DRG system with regard to MDC 16, even in its 8th generation. A comparison of the reimbursement level with the level of costs within MDC 16 reveals an even more dramatic result. An unpublished analysis of the German University Hospital Consortium (Verband der Universitätsklinika Deutschlands, VUD) showed for 10 university hospitals that the medical DRGs Q60 and Q61 are not

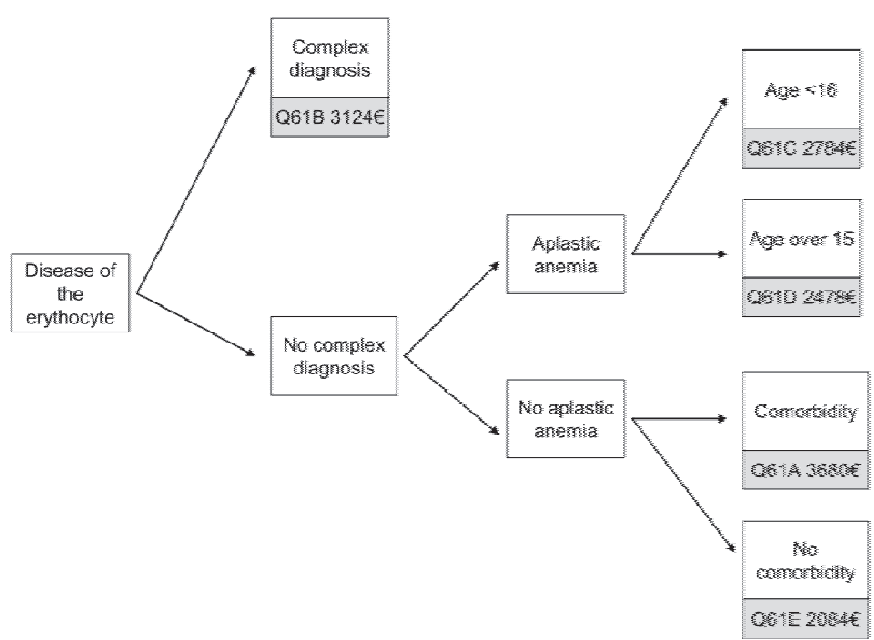

Fig. 4. MDC 16 - Diseases of the Erythrocytes, simplified (base rate EUR 2,935.00).

Table 2. Complex diagnoses eligible for DRG Q61B

Vitamin B12 deficiency anaemia (ICD D51.-)

Sickle cell anaemia with crisis (ICD D57.0)

Drug-induced autoimmune haemolytic anaemia (ICD D59.0)

Other autoimmune haemolytic anaemia (ICD D59.1)

ABO incompatibility reaction (ICD T80.3)

cost-covering at all. Indeed the funding gap was $-24 \%$ overall, with Q61D at $-55 \%$ and Q61A at $-44 \%$ incurring the most dramatic losses. We believe that this discrepancy is due to a mixture of drug-induced aplastic anaemia accounting for the low cost cases ( $76 \%$ of cases put into the calculation) and severe aplastic anaemia accounting for the high cost cases typically being treated at university hospitals ( $5 \%$ of cases). It may be possible that patients with severe aplastic anaemia should be allocated to the complex diagnosis group.

\section{Challenge 2: Trying to Adequately Remunerate the Treatment of All Patients Presenting with a Concomitant Diagnosis Typical for Transfusion Medicine}

The majority of patients requiring transfusion medicine and hemotherapy do not visit the hospital due to a diagnosis primarily from this field of medicine. In fact, the need for transfusion is almost ubiquitous, and in almost all DRGs transfusion can be found. In an analysis carried out in 60 university hospitals in the USA in 1995, Jefferies et al. [5] showed that 471 of 486 DRGs had identifiable blood costs. The highest mean costs could be found in the DRG for bone marrow transplant (DRG 481) with USD 6,183.00, followed by liver transplant (DRG 480, USD 5,548.00) and acute leukaemia 
Table 3. Examples of the impact of PCCL 4 on remuneration for oncologic DRGs with conservative treatment such as chemotherapy (base rate EUR 2,950.00)

\begin{tabular}{lll}
\hline DRG & $\begin{array}{l}\text { PCCL 0-3 } \\
\text { (remuneration, EUR) }\end{array}$ & $\begin{array}{l}\text { PCCL 4 } \\
\text { (remuneration, EUR) }\end{array}$ \\
\hline B66 brain cancer & B66D $(2,107.00)$ & B66B $(3,757.00)$ \\
D60 ENT cancer & D60C $(1,767.00)$ & D60B $(2,184.00)$ \\
E71 thorax cancer & E71B $(1,714.00)$ & E71A $(3,322.00)$ \\
G60 GI cancer & G60B $(1,162.00)$ & G60A $(1,911.00)$ \\
H61 pancreas cancer & H61B $(1,914.00)$ & H61A $(3,369.00)$ \\
I65 soft tissue cancer & I65C $(2,322.00)$ & I65B $(3,337.00)$ \\
J62 breast cancer & J62B $(1,702.00)$ & J62A $(3,349.00)$ \\
L62 genitourinary cancer & L62B $(1,576.00)$ & L62A $(3,273.00)$ \\
M60 cancer of the male genitals & M60C $(1,644.00)$ & M60A $(3,493.00)$ \\
N60 cancer of the female genitals & N60B $(1,661.00)$ & N60A $(3956.00)$ \\
\hline ENT = Ear nose throat; GI = gastrointestinal. & \\
\hline
\end{tabular}

(DRG 473, USD 4,427.00). The transfusion costs represented a small portion $(<1 \%)$ of the total hospital costs. Syrjala et al. [16] analyzed the transfusion practice in different DRGs in a university hospital in Finland. They found that most of the DRGs had blood costs but that half of the total costs could be found in 13 different DRGs. Among them, acute leukaemia (DRG 473) was the most blood consuming with 7.3 RBC and 45 platelet units transfused per patient (total costs per transfused patient: EUR 3,689.00). Other DRGs with high blood consumption were bone marrow transplant (DRG 481), lymphoma and non-acute leukaemia (DRG 404), tracheostomy (DRG 483), and liver transplant (DRG 480). The departments with the highest transfusion costs were surgery (39\%), internal medicine $(34 \%)$, and paediatrics $(19 \%)$. For Germany, national data is published on a more aggregate level and is more difficult to interpret due to the fact that the need for transfusion is not coded by 1 code only. Different codes exist depending on the number of transfusion units needed.

Of the 15,559,359 hospitalizations in Germany in 2007, only 116,093 had a principal diagnosis from chapter III of the ICD-10 classification (diseases of the blood and blood-forming organs, and certain disorders involving the immune mechanism). As discussed in the previous paragraph, the diagnoses of chapter III can usually be found in the MDC 16. For all other DRGs that are not part of MDC 16, in no case a diagnosis from the field of transfusion medicine or a procedure from the field of hemotherapy will immediately influence the grouper result. Usually, the rationale as to why a distinct DRG is targeted is not dependent on whether transfusion medicine was necessary or not. This does not mean that transfusion medicine and hemotherapy remain totally unconsidered in the remuneration system beyond MDC 16. To make it possible to accent pre-existing medical conditions and concomitant complications, 3 major principles have been introduced in the G-DRG system: i) Diagnoses from the field of transfusion medicine usually increase the comorbidity and complexity level (CCL); ii) Procedures from the field of hemotherapy can be used in a so-called function which is a pre- defined combination of different procedure codes; iii) Procedures may be directly remunerated by ZE.

A patient's level of comorbidity and complexity (Patient Clinical Complexity Level, PCCL) can range in value from 0 up to 4. PCCL calculation is based on an algorithm developed by Dr. Xichuan Zhang in the CCL refinement project in Australia [17]. Comorbidities and complications (CC) are concomitant diagnoses that usually cause higher resource consumption. A total of 3,459 ICD-10 codes have been marked as CC in the G-DRG 2010 system. A CCL is allocated to every $\mathrm{CC}$, and CCL can have values from 1 to 6 . A logarithmic formula combines all CCLs to a calculated value, and after rounding the PCCL is available. PCCL is a whole number between 0 (no comorbidity) and 4 (highest level of comorbidity). PCCL is widely used as a splitting criterion to identify cases with higher resource consumption. Usually PCCL levels of 4 have to be reached to change the DRG split and to obtain a higher remuneration.

In the field of transfusion medicine, there are numerous diagnoses which increase the PCCL level. The number of the diagnoses usually used in hemotherapy without a CCL is low. From the chapter of nutritional anaemias (D50-D53) only vitamin B12 deficiency anaemias (D51.-) are excluded. Among haemolytic anaemias (D55-D59), all diagnoses show a CCL except the anaemias due to enzyme disorders (D55) and other hereditary haemolytic anaemias (D58). For aplastic anaemias, the exclusion list comprises ICD-10 D63.8 (anaemia in other chronic diseases classified elsewhere), ICD-10 D64.4 (congenital dyserythropoietic anaemia), ICD-10 D64.8 (other specified anaemias), and ICD-10 D64.9 (anaemia, unspecified). For coagulation defects, purpura, and other hemorrhagic conditions (D65-D69), a CCL has been defined for all diagnoses. Thus, all coagulation defects are of PCCL relevance.

As an example, table 3 shows the impact of PCCL 4 on DRG remuneration for the field of oncology in which hemotherapy is common in the author's opinion. Whereas the median remuneration for patients with a PCCL of 0-3 ranges around EUR 1,700.00, the rate for the treatment of patients 
Table 4. Examples of the impact of the function 'complex constellation' on remuneration for intensive care DRGs or brain cancer DRGs with conservative treatment such as chemotherapy (base rate EUR 2,950.00)

\begin{tabular}{lll}
\hline DRG & $\begin{array}{l}\text { Without complex constellation } \\
\text { (remuneration, EUR) }\end{array}$ & $\begin{array}{l}\text { With complex constellation } \\
\text { (remuneration, EUR) }\end{array}$ \\
\hline $\begin{array}{l}\text { A13 mechanical ventilation } \\
>95 \mathrm{~h} \text { and up to }<250 \mathrm{~h}\end{array}$ & A13G $(10,912.00)$ & A13E $(20,134.00)$ \\
B66 brain cancer & B66D $(2,107.00)$ & B66A $(8,162.00)$ \\
\hline
\end{tabular}

Table 5. Additional remuneration fees (ZE) in transfusion medicine and hemotherapy showing the lowest and the highest amounts paid for national prices in 2010

\begin{tabular}{ll}
\hline ZE & Price list range, EUR \\
\hline ZE 36 plasmapheresis & $1,305.00(\times 1)-67,874.00(>\times 49)$ \\
ZE 84 thrombocyte concentrate (apheresis) & $458.00(\times 1)-55,002.00(>\times 117)$ \\
ZE94 thrombocyte concentrate (non-apheresis) & $535.00(\times 2)-34,778.00(>\times 127)$ \\
ZE107 packed red cells & $608.00(\times 6)-22,630.00(>\times 279)$ \\
ZE108 thrombocyte concentrate, patient-specific & $490.00(\times 1)-35,257.00(>\times 70)$ \\
ZE2010-15 cell apheresis & $589.00(\times 1)$ \\
ZE2010-34 $4^{\text {a }}$ granulocyte concentrate & $750.00(\times 1)-$ XXX $(>\times 20)$ \\
ZE2010-35 acquisition of stem cells & $10,200.00($ Germany $)-25,830.00$ (overseas) \\
\hline
\end{tabular}

${ }^{a}$ No national price defined; listed price represents the price list of the author's institution. with a PPCL of 4 will be approximately EUR 3,300.00. Of course this duplication of remuneration is not triggered by hemotherapy and transfusion medicine only, but reflects the higher resource consumption for the treatment of these multimorbid patients in general.

Procedures from the field of hemotherapy can trigger a socalled function that may cause a shift to a more valuable DRGs and result in higher remuneration. Functions have very complex definitions. To get a function activated, more than 1 condition must prevail. The most prominent and common function in the G-DRG system is the term 'complex constellation'. For a complex constellation, at least 2 platelet concentrates or at least $11 \mathrm{PRC}$ have to be given (condition 1), and patients have to undergo mechanical ventilation for $>48 \mathrm{~h}$ or be diagnosed with disseminated intravascular coagulation (DIC) or receive specific drainage treatment / pacemaker treatment / invasive monitoring / radiation treatment or undergo hemodialysis or resuscitation (condition 2). The exact definition of complex constellation lists up over 20 different combinations [10]. Table 4 shows the impact of the function 'complex constellation' on DRG remuneration for brain tumours and for DRG A13, a common DRG for patients requiring intensive care with mechanical ventilation for 4-10 days.Procedures may be remunerated directly by ZE (additional remuneration fees). The ZE system was introduced in 2005 after 2 years of testing of the G-DRG. It was observed that a major reason for cost variability could be found in specific expensive items such as implants, drugs, or blood products. By separating these expensive items from the lump sum and generating a pricelist for ZE, a much more homogeneous cost distribution in the DRG itself could be achieved. ZE price lists follow a national standard and are updated annually. Usually the ZE price is predefined and not negotiable for the hospital. For very rare procedures, however, hospitals can negotiate individual $\mathrm{ZE}$ prices. For the field of hemotherapy and transfusion medicine, fixed price $\mathrm{ZE}$ can be found for plasmapheresis (ZE 36) and the application of thrombocyte concentrates generated by apheresis (ZE 84), thrombocyte concentrates (ZE 94), PRC (ZE 107) and patient-specific thrombocyte concentrates (ZE 108). ZE with a price that can be negotiated by the hospital exist for cell apheresis (ZE201015), the application of granulocyte concentrates (ZE2010-34), and the acquisition of hematopoietic stem cells (ZE2010-35). Table 5 lists the lowest and the highest price for the named procedures. Some procedures are not paid additionally unless a minimum amount is administered. For example, for PRC, at least 6 PRC have to be given for children under the age of 15 and at least 16 PRC have to be given for adolescents over 14 and adults to trigger a $\mathrm{ZE}$ remuneration.

What can be done to improve the remuneration for transfusions in Germany? While, as outlined above, the principle tools to document the additional costs are provided within the G-DRG system (PCCL and complex constellation as split criterion, ZE), they are probably not used as well as they could be. The main problem is the profound and exact documentation of all costs associated with transfusion. The costs that are used to remunerate transfusions in the DRG system are probably based almost solely on acquisition costs. Infrastructural costs for transport and storage and handling of the product are likely not associated with the case costs. Furthermore, laboratory costs (e.g. cross-matching) and labour costs for performing and documenting the transfusion are likely not included in the calculation data used by the InEK to calculate DRG and ZE. Therefore, every endeavour should be made to associate transfusion costs with the case. This can only be achieved by close collaboration between the clinical depart- 
Table 6. Candidates for a NUB (New Diagnostic and Treatment Methods) fee relevant to hemotherapy and transfusion medicine in 2010
NUB11 romiplostim

NUB32 plerixafor

NUB525 eltrombopag ${ }^{\mathrm{a}}$

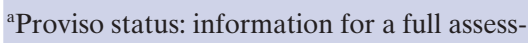
ment of this method was insufficient.

ment, the department of transfusion medicine, and the administration. Once this has been done, it should be reflected in increased DRG remunerations and increased ZE.

\section{Challenge 3: How Can We Assure that Medical Progress Is Rapidly Implemented into Clinical Routine and Not Obstructed by Financial Restraints?}

The G-DRG system was designed to be adaptive. It is annually revised by a cost calculation based on real life cost data. As the annual calculation is always based on the treatment data of the preceding year and is calculated and published for the subsequent year, an inevitable 2-year gap between expenditure and remuneration occurs. Innovations that are consumed by the hospital in this 2-year period will neither be covered by G-DRG lump sums nor by ZE. To bridge this innovation gap, the New Diagnostic and Treatment Methods (Neue Untersuchungs- und Behandlungsmethoden, NUB) system was intro-

duced in 2006. NUBs can be negotiated immediately between the hospital willing to use the new innovation and the sick fund. An exclusive NUB list is issued annually by the InEK. Only procedures listed on this NUB-list can be negotiated, after an application to use a specific method/drug has been issued by the individual hospital to the InEK [18]. Table 6 lists the NUB candidates from the field of hemotherapy and transfusion medicine in 2010. The NUB principle allows for remuneration of a drug or method immediately after its introduction. For blood products, no NUB currently exists. As only new drugs and treatment methods fulfil the requirements of NUB, transfusion of traditional blood products (PRC, platelets) does not qualify. One NUB that is associated with transfusion medicine is plerixafor which is used for the collection of peripheral blood stem cells in patients failing to respond to granulocyte colony-stimulating factor. This new drug has been granted NUB status 1 , meaning additional remuneration can be negotiated with the health insurance funds. The exact amount of money paid is also subject to these negotiations. Hence, the NUB procedure provides for adequate remuneration for these patients. Patient-specific cellular therapies are another example of a transfusion medicine product which is new, expensive, and therefore eligible for the NUB process.

\section{Disclosure Statement}

The authors did not provide a conflict of interest statement.

\section{References}

1 Amin M, Fergusson D, et al.: The societal unit cost of allogenic red blood cells and red blood cell transfusion in Canada. Transfusion 2004;44:14791486.

2 InEK GmbH: Abschlussbericht zur Weiterentwicklung des G-DRG-Systems für 2010 (Final Report on the Further Development of the G-DRGSystem for the Year 2010). www.g-drg.de/cms/ index.php/inek_site_de/G-DRG-System_2010/ Abschlussbericht_zur_Weiterentwicklung_des_GDRG-Systems_und_Report_Browser/Abschlussbericht_zur_Weiterentwicklung_des_G-DRGSystems_fuer_2010.

$\checkmark 3$ Goodman C, Chan S, et al.: Ensuring blood safety and availability in the US: technological advances, costs, and challenges to payment - final report. Transfusion 2003;43(suppl 8):3S-46S.

$\checkmark 4$ Goodnough LT, Brecher ME, et al.: Transfusion medicine. First of two parts - blood transfusion. N Engl J Med 1999;340:438-447.

5 Jefferies LC, Sachais BS, et al.: Blood transfusion costs by diagnosis-related groups in 60 university hospitals in 1995. Transfusion 2001;41:522-529.
6 Shander A, Hofmann A, et al.: Estimating the cost of blood: past, present, and future directions. Best Pract Res Clin Anaesthesiol 2007;21:271-289.

7 No authors listed: The cost of blood: multidisciplinary consensus conference for a standard methodology. Transfus Med Rev 2005;19:66-78.

8 Centers for Medicare and Medicaid Services (CMS), HHS: Medicare program; changes to the hospital inpatient prospective payment system for acute care hospitals and fiscal year 2010 rates; and changes to the long-term care hospital prospective payment system and rate years 2010 and 2009 rates. Final rules and interim final rule with comment period. Fed Regist 2009;74:43753-4236.

9 Glocker S, Loskamp N, et al.: [Evaluation of flatrate payment in radiation oncology. German experience with disease-related groups for inpatient funding in radiation oncology]. Strahlenther Onkol 2006;182:305-311.

10 InEK GmbH: German Diagnosis Relates Groups Version 2010. Definitionshandbuch.

11 InEK GmbH: Deutsche Kodierrichtlinien 2010 (German Coding Guidelines 2010). www.g-drg.de/ cms/index.php/inek_site_de/content/view/full/2347.
12 Forbes JM, Anderson MD, et al.: Blood transfusion costs: a multicenter study. Transfusion 1991; 31:318-323.

13 Cantor SB, Hudson DV Jr, et al.: Costs of blood transfusion: a process-flow analysis. J Clin Oncol 1998;16:2364-2370.

14 Shander A, Hofmann A, et al.: Activity-based costs of blood transfusions in surgical patients at four hospitals. Transfusion 2010;50:753-765.

15 Volkmer BG, Petschl S, et al.: [Relevance of hospitals participating in the DRG calculation for the development of the G-DRG system]. Urologe A 2008;47:866-872, 874.

16 Syrjala MT, Kytoniemi I, et al.: Transfusion practice in Helsinki University Central Hospital: an analysis of diagnosis-related groups (DRG). Transfus Med 2001;11:423-431.

17 Antioch K, Zhang X: Organ transplant AN-DRGs: modifying the exceptions hierarchy in casemix classification. Aust Health Rev 2000;23:137-152.

18 Brechmann T Schmiegel W: [Procedure of implementation of new methods of examination and treatment in the G-DRG system (NUB procedure)]. Med Klin (Munich) 2007;102:683-684. 